\title{
Preface
}

\section{Risk Factors in Atrial \\ Fibrillation: Appraisal of Atrial Fibrillation Risk Stratification}

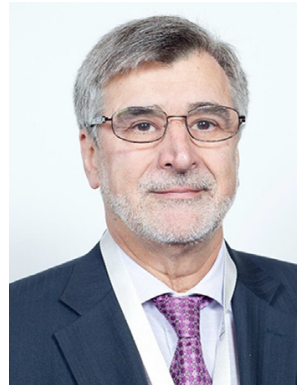

Mohammad Shenasa, MD

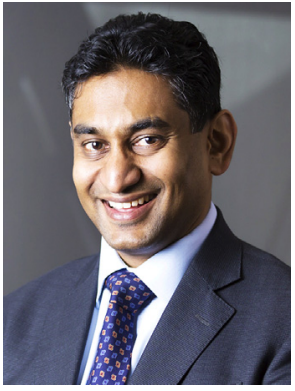

Prashanthan Sanders, MBBS, PhD

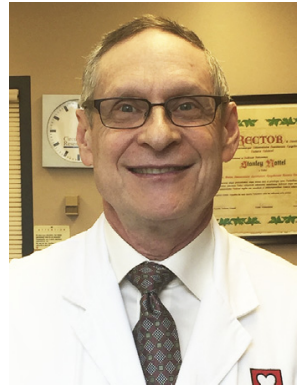

Stanley Nattel, MD

Editors

Atrial fibrillation (AF) remains the most common sustained arrhythmia encountered in clinical practice, with an increasing incidence related to increasing survival of patients with AF-promoting comorbidities as well as population aging. Accordingly, the prevalence of important AF-associated morbidities, like stroke and heart failure, is increasing, along with the related social and economical burden. Despite significant progress in understanding the pathophysiologic mechanisms of $\mathrm{AF}$ on one hand and the advances in the catheter ablation of AF on the other, the present management options are still far from perfect.

Comprehensive management of $\mathrm{AF}$ requires a multidisciplinary approach beyond catheter ablation.

Over the past couple of decades, a large body of evidence has demonstrated that multiple risk factors predispose to AF occurrence. Among these are well-established risk factors, like genetic predisposition, hypertension, heart failure, diabetes, obesity, sleep apnea, cigarette smoking, pulmonary disease, cardiomyopathies, endocrine abnormalities, valvular heart disease, and coronary artery disease. There are also some novel risk factors associated with $\mathrm{AF}$, such as alcohol consumption, air pollution, social and recreational habits, and infectious diseases, like COVID-19.

Recent work has demonstrated that the effective targeting of AF risk factors with a multidisciplinary approach is a potentially important therapeutic intervention, with the potential to reduce AF burden, increase the long-term success of ablation therapy, and in some cases, even obviate ablation.

Although these risk factors are individually discussed in several textbooks and articles, a collective and comprehensive text is overdue.

In this issue of Cardiac Electrophysiology Clinics, we dive into the depth of AF risk factors by inviting leaders in each area to provide their current perspective on the state-of-the-art.

We have arranged the Table of Contents according to specific risk factors.

We are confident that this first collective text on the management of AF risk factors will be useful to those who take care of patients with AF. 
We wish to thank Ms Mona Soleimanieh for her superb assistance in finishing this project.

Mohammad Shenasa, MD Heart and Rhythm Medical Group 18324 Twin Creeks Road Monte Sereno, CA 95030, USA

Prashanthan Sanders, MBBS, PhD Centre for Heart Rhythm Disorders South Australian Health and Medical Research Institute University of Adelaide and Royal Adelaide Hospital Adelaide, Australia
Stanley Nattel, MD Montreal Heart Institute and Université de Montréal Montréal, Quebec, Canada

Department of Pharmacology and Therapeutics McGill University 3655 Promenade Sir William Osler Montreal, Quebec H3G 1Y6, Canada

E-mail addresses:

mohammad.shenasa@gmail.com (M. Shenasa) prash.sanders@adelaide.edu.au (P. Sanders) stanleynattel@gmail.com (S. Nattel) 